\title{
miR-202 functions as a tumor suppressor in hepatocellular carcinoma by targeting HK2
}

\author{
JIANGANG WANG, JILI CHEN, FANG SUN, ZHIWEI WANG, WENFANG XU, \\ YAFENG YU, FENG DING and HUAJIANG SHEN
}

Department of Liver Disease, Affiliated Hospital of Shaoxing University, Shaoxing, Zhejiang 312000, P.R. China

Received November 17, 2018; Accepted August 1, 2019

DOI: $10.3892 / \mathrm{ol} .2020 .11334$

\begin{abstract}
Recent evidence has suggested that microRNAs (miRNAs) can participate in metabolic reprogramming. Additionally, aerobic glycolysis is associated with tumor progression in hepatocellular carcinoma (HCC). In the present study, miRNA (miR)-202 expression levels were found to be significantly lower in HCC tissues compared with the corresponding adjacent non-cancerous tissue samples using reverse transcription-quantitative PCR analysis in 56 patients with HCC. Lower miR-202 expression levels were identified to be associated with tumor size, vascular invasion, Tumor, Node and Metastasis stages and poor overall survival rates in patients with HCC. In vitro, upregulation of miR-202 expression was revealed to significantly suppress the cell glucose uptake, lactate production and cell proliferation in liver cancer cells. In addition, dual luciferase reporter analysis and western blot assays suggested that hexokinase 2 (HK2) was a direct target of miR-202. Upregulation of miR-202 expression could inhibit cell proliferation by regulating HK2 expression in HCC. Therefore, the results from the present study suggested that miR-202 may serve as a potential target for HCC treatment.
\end{abstract}

\section{Introduction}

Hepatocellular carcinoma (HCC) was the fifth most common malignancy and was the second-most frequent cause of cancer-associated mortality worldwide in 2012 (1). Every year, 780,000 new cases of HCC are diagnosed and 745,000 mortalities are due to HCC tumor progression (2). Despite the advancements in therapeutic strategies, including improvements in surgery and radio-chemotherapy regimens for patients with $\mathrm{HCC}$, the 5-year survival rate remains low

Correspondence to: Dr Huajiang Shen, Department of Liver Disease, Affiliated Hospital of Shaoxing University, 999 Zhongxing South Road, Shaoxing, Zhejiang 312000, P.R. China

E-mail: jqhf27563799t@163.com

Key words: hepatocellular carcinoma, microRNA-202, hexokinase 2, cell proliferation, glycolysis due to the high recurrence and metastasis of the disease (3). Therefore, identification of novel and effective therapies is of prime importance.

Cancer cells exhibit reprogrammed metabolism, and present aerobic glycolysis, which is a phenomenon known as the Warburg effect $(4,5)$. The Warburg effect is one of the major metabolic changes in tumor cells, which is characterized by an increased glucose uptake and lactate production in the presence of oxygen $(4,5)$. MicroRNAs (miRNAs) have previously been demonstrated to be involved in energy metabolism (6), for example, the mineralocorticoid receptor suppresses the progression of cancer and the Warburg effect via modulation of the miRNA (miR)-338-3p/pyruvate kinase axis in HCC (7). miR-199a maturation is suppressed by RNA-binding protein human antigen $\mathrm{R}$, which is crucial for the hypoxia-induced glycolytic switch in HCC (8). STAT3-mediated activation of miR-23a suppresses gluconeogenesis in HCC by downregulating glucose-6-phosphatase and peroxisome proliferator-activated receptor $\gamma$, coactivator $1 \alpha$ (9). However, the roles of miR-125 in HCC progression and glycolysis remain unknown.

The present study suggested that miR-202 expression was lower in HCC tissues, and lower miR-202 expression was associated with poor prognosis in patients with HCC. In vitro, miR-202 was found to suppress cell glucose uptake, lactate production and cell proliferation. Furthermore, it was identified that hexokinase 2 (HK2) was a target of miR-202. miR-202 inhibited cell proliferation and cells glycolysis by targeting HK2 in HCC. Therefore, these results suggested that miR-202 may serve as a potential therapeutic target for treating HCC.

\section{Materials and methods}

Clinical tissue samples. A total of 56 patients with HCC including 43 males and 13 females with a mean age of $55 \pm 8.17$ years (range, $32-78$ years) were recruited in to the present study. Paired fresh HCC tissues and corresponding adjacent $(2 \mathrm{~cm})$ non-cancerous tissue samples (the distance between the samples of the HCC tissues and the adjacent non-cancerous tissues is $2 \mathrm{~cm}$ ) were obtained from patients with HCC undergoing hepatectomy at the Department of Liver Disease, Affiliated Hospital of Shaoxing University (Shaoxing, China) from March 2008 to February 2015. The tissue samples were confirmed as HCC by histopathological 
examination. None of the patients in the present study received chemotherapy or radiation therapy prior to surgery. Following surgery, the sample was stored at $-80^{\circ} \mathrm{C}$ until further use. The present study was approved by the Institutional Ethics Committee of Affiliated Hospital of Shaoxing University. Written informed consent was obtained from all patients that participated in the present study.

Cell line culture. Human liver cancer cells, including HepG2, 97-L, Hep3B, Huh-7 and a hepatocellular cell line (THLE-3) were purchased from the cell bank of type culture collection at the Chinese Academy of Sciences (Shanghai, China). All cells were cultured in DMEM (HyClone; GE Healthcare Life Sciences) and supplemented with 10\% FBS (Gibco; Thermo Fisher Scientific, Inc.), $100 \mathrm{U} / \mathrm{ml}$ penicillin and $100 \mathrm{mg} / \mathrm{ml}$ streptomycin (Gibco; Thermo Fisher Scientific, Inc.) at $37^{\circ} \mathrm{C}$ in a humidified incubator with $5 \% \mathrm{CO}_{2}$.

Reverse transcription-quantitative PCR (RT-qPCR) analysis. Total RNA isolation from HCC tissues and liver cancer cell lines was performed using TRIzol ${ }^{\circledR}$ (Invitrogen; Thermo Fisher Scientific, Inc.). The cDNA was reversed transcribed using RNA with a reverse transcription kit (Applied Biosystems; Thermo Fisher Scientific, Inc.) using the following cnditions: $18^{\circ} \mathrm{C}$ for $30 \mathrm{~min}, 42^{\circ} \mathrm{C}$ for $30 \mathrm{~min}$ and $90^{\circ} \mathrm{C}$ for $5 \mathrm{~min}$. RT-qPCR was performed using an ABI 7500 real-time system with SYBR Premix Ex Taq kit (Takara Biotechnology Co., Ltd.) according to the manufacturer's protocol. The following conditions were used: Initial denaturation at $95^{\circ} \mathrm{C}$ for $5 \mathrm{~min}$, 30 cycles of denaturation at $94^{\circ} \mathrm{C}$ for $30 \mathrm{sec}$, annealing at $56^{\circ} \mathrm{C}$ $\left(58^{\circ} \mathrm{C}\right.$ was used for GAPDH) for $30 \mathrm{sec}$, and extension at $72^{\circ} \mathrm{C}$ for $30 \mathrm{sec}$. Data were analyzed using the $2^{-\Delta \Delta \mathrm{Cq}}$ method (10). GAPDH was used as an internal control. The forward and reverse sequences were as follows: GAPDH: forward, 5'-GAC TCATGACCACAGTCCATGC-3'; reverse, 5'-AGAGGCAGG GATGATGTTCTG-3'; HK2: forward: 5'-CCACAGGTC ATCATAGTTCC-3'; reverse, 5'-GGCACCCAGCACAAT GAAG-3'; miR-202: forward, 5'-CTCCAGAGAGAUAGU AGAGCCT-3'; reverse, 5'-CTCAACCAATCACCTGGC ACAGA-3'; U6: forward, 5'-CTCGCTTCGGCAGCACA-3'; reverse, 5'-AACGCTTCACGAATTTGCGT-3'. GAPDH was used as the endogenous control for normalization of the mRNA quantification and U6 was used as the endogenous control for normalization of the miRNA quantification.

Cell transfection. The miR-202 mimic (50 nM), miR-202 inhibitor $(50 \mathrm{nM})$ and miRNA negative control $(\mathrm{NC} ; 50 \mathrm{nM})$ and small interfering (si)-negative control $(50 \mathrm{nM})$ or si-HK2 (50 $\mathrm{nM}$ ) were designed by and purchased from Shanghai GenePharma Co., Ltd. Cells were seeded in 6-well plates until they reached $80-90 \%$ confluence and were then transfected using Lipofectamine ${ }^{\circledR}$ 3,000 reagent (Invitrogen; Thermo Fisher Scientific, Inc.) according to the manufacturer's protocol. Cells were harvested $48 \mathrm{~h}$ after cell transfection.

Cell proliferation assay. Cells were seeded into 96-well plates (3,000 cells/well) and then transfected with miR-202 mimic, miR-202 inhibitor or miR-NC. Following cell transfection at $0,24,48,72$ and $96 \mathrm{~h}$, cell proliferation was detected using a Cell Counting Kit 8 (CCK8; Dojindo Molecular Technologies,
Inc.) according to the manufacturer's protocol. Briefly, cells were incubated with $10 \mu \mathrm{lCCK} 8$ reagents for $2 \mathrm{~h}$ at $37^{\circ} \mathrm{C}$. The optical density value was detected at $450 \mathrm{~nm}$ using an ELISA reader (Bio-Rad Laboratories, Inc.).

Target prediction. The predicted binding sites between miR-202 and HK2 were obtained using Miranda online software 2010 (http://www.microrna.org/microrna/home.do; August 2010 release).

Glucose uptake assay. Cells were seeded in 6-well plates and transfected with miR-202 mimic (50 nM), miR-202 inhibitor (50 nM) or miR-NC (50 nM). At 48 h, 97-L and Huh7 cells were cultured with serum-starved and glucose-free medium (DMEM; HyClone; GE Healthcare Life Sciences). Cells were then treated with $50 \mathrm{mM}$ 2-[N-(7-nitrobenz-2-oxa-1,b 3-diazol-4-yl) amino]-2-deoxy-D-glucose (Invitrogen; Thermo Fisher Scientific, Inc.) for $1 \mathrm{~h}$ at $37^{\circ} \mathrm{C}$. Glucose uptake was quantified using a flow cytometer (Pathway 855 Bioimaging system; BD Pathway ${ }^{\mathrm{TM}} 800$ Series Software; BD Bioscience).

Lactate production assay. Cells were seeded in 6-well plates and transfected with miR-202 mimic, miR-202 inhibitor or miR-NC, as aforementioned. At $48 \mathrm{~h}$, culture medium was removed from cells and lactate concentration was determined using lactate test strips and an Accutrend Lactate analyzer (Accutrend Lactate; Roche Diagnostics). Results were normalized to the cell number in the negative control group.

Western blot analysis. Proteins were extracted using a RIPA lysis buffer (Beyotime Institute of Biotechnology). The protein concentration was determined using a bicinchoninic acid assay protein assay kit (Pierce; Thermo Fisher Scientific, Inc.). A total of $50 \mu \mathrm{g}$ extracted protein samples were separated via SDS-PAGE on $10 \%$ gels and transferred to a PVDF membrane (EMD Millipore). The membranes were blocked in 5\% skimmed milk at room temperature for $1 \mathrm{~h}$ and incubated with primary antibodies anti-HK2 (1:1,000; cat. no. sc-6521; Santa Cruz Biotechnology, Inc.) at $4^{\circ} \mathrm{C}$ overnight. The membranes were then incubated with the corresponding horseradish peroxidase-conjugated goat anti-rabbit IgG secondary antibodies (1:1,000; cat. no. A0208; Beyotime Institute of Biotechnology) for $1 \mathrm{~h}$ at room temperature. The protein bands were detected using an ECL kit (Beyotime Institute of Biotechnology).

Dual luciferase reporter assay. The 97L and Huh7 $(10,000 /$ well) cells were incubated in 24-well plates for the dual-luciferase reporter assay (Promega Corporation) at $37^{\circ} \mathrm{C}$. The wild- or mutant-type of 3'-untranslated region (3'-UTR) of HK2 was inserted into the psiCHECK2 vector (Invitrogen; Thermo Fisher Scientific, Inc.). Then, wild- or mutant-type 3-UTR HK2 and miR-202 mimic were transfected with Lipofectamine $^{\circledR} 3000$ reagent (Invitrogen; Thermo Fisher Scientific, Inc.) according to the manufacturer's protocol into 97L and Huh7 cells. After 48 h, the dual luciferase reporter assay system (Promega Corporation) was applied to measure luciferase activities and the results were normalized to Renilla luciferase activity. 
Statistical analysis. Data are presented as the mean \pm SD. Differences were compared using a paired Student's t-test or one-way ANOVA and Student-Newman-Keuls was used as a post hoc test following the ANOVA. The patients were divided into two groups: i) High miR-202 expression; and ii) low miR-202 expression groups, according to the median expression of miR-202 in HCC tissue samples. The association between the clinicopathological features of HCC and miR-202 was calculated using the $\chi^{2}$ test. The overall survival rates and survival differences were detected using univariate analysis and Kaplan-Meier method with the log-rank test. $\mathrm{P}<0.05$ was considered to indicate a statistically significant result.

\section{Results}

miR-202 expression is downregulated in HCC tissue samples and liver cancer cells. In the present study, the expression levels of miR-202 were detected in HCC tissue samples and the corresponding adjacent non-cancerous tissue samples using RT-qPCR analyses. The results indicated that miR-202 expression was significantly downregulated in HCC tissue samples compared with the corresponding adjacent non-cancerous tissue samples $(\mathrm{P}<0.05$; Fig. $1 \mathrm{~A})$. Furthermore, the RT-qPCR results revealed that miR-202 expression was dramatically downregulated in several liver cancer cells compared with THLE-3 cells ( $\mathrm{P}<0.05$; Fig. 1B). The patients were divided into two groups: i) High miR-202 expression; and ii) low miR-202 expression groups, according to the median expression (0.45-fold) of miR-202 in HCC tissue samples. The $\chi^{2}$ analysis was applied to detect the potential associations between miR-202 expression and the clinical characteristics. The results suggested that miR-202 expression was significantly associated with tumor size, vascular invasion and Tumor, Node and Metastasis (TNM) stage (11) of the patients with HCC (all $\mathrm{P}<0.05$; Table I). However, there was no association with age, sex, differentiation and AFP level (all $\mathrm{P}>0.05$; Table I). Furthermore, a survival plot was calculated using the Kaplan-Meier method and Log-rank test between the high $(n=27)$ and low $(n=29)$ median miR-202 expression groups. The results indicated that patients with higher miR-202 expression levels exhibited longer survival rates compared with patients with lower miR-202 expression levels $(\mathrm{P}<0.05$; Fig. 1C), suggesting that lower expression levels of miR-202 contributed to the development of HCC, and the expression level of miR-202 may serve as a predictor of HCC.

miR-202 inhibits cell proliferation and cell glycolysis in HCC. The Warburg effect is characterized by an increase in glucose uptake and lactate production in the presence of oxygen (6). Whether miR-202 expression affected cell proliferation and glycolysis in HCC cells was further investigated in the present study. The gain-of-function and loss-of-function assays were performed by transfecting miR-202 mimic or miR-202 inhibitor into 97-L and Huh7 cells due their higher and lower miR-202 expression levels in numerous liver cancer cell lines. Importantly, both miR-202 mimic and inhibitor significantly affected the expression level of miR-202 (Fig. 2A and B). Compared with the negative controls, the CCK8 results revealed that transfection of miR-202 mimic in 97-L and Huh7 cells significantly inhibited cell proliferation, while
Table I. Association between miR-202 expression and clinicopathological parameters in 56 patients with hepatocellular carcinoma.

\begin{tabular}{|c|c|c|c|c|}
\hline \multirow[b]{2}{*}{$\begin{array}{l}\text { Clinicopathological } \\
\text { parameters }\end{array}$} & \multirow[b]{2}{*}{ Total } & \multicolumn{2}{|c|}{ miR-202 expression } & \multirow[b]{2}{*}{ P-value } \\
\hline & & $\begin{array}{l}\text { High } \\
(n=27)\end{array}$ & $\begin{array}{c}\text { Low } \\
(n=29)\end{array}$ & \\
\hline Age, years & & & & 0.643 \\
\hline$\leq 55$ & 41 & 19 & 22 & \\
\hline$>55$ & 15 & 8 & 7 & \\
\hline Sex & & & & 0.422 \\
\hline Male & 43 & 22 & 21 & \\
\hline Female & 13 & 5 & 8 & \\
\hline Tumor size, $\mathrm{cm}$ & & & & 0.015 \\
\hline$<5$ & 30 & 19 & 11 & \\
\hline$\geq 5$ & 26 & 8 & 18 & \\
\hline Differentiation & & & & 0.672 \\
\hline Well and moderately & 40 & 20 & 20 & \\
\hline Poor & 16 & 7 & 9 & \\
\hline $\mathrm{AFP}(\mathrm{ng} / \mathrm{ml})$ & & & & 0.336 \\
\hline$<400$ & 18 & 7 & 11 & \\
\hline$\geq 400$ & 38 & 20 & 18 & \\
\hline Vascular invasion & & & & 0.012 \\
\hline Negative & 34 & 21 & 13 & \\
\hline Positive & 22 & 6 & 16 & \\
\hline TNM stage & & & & 0.035 \\
\hline I-II & 38 & 22 & 16 & \\
\hline III-IV & 18 & 5 & 13 & \\
\hline
\end{tabular}

miR, microRNA; AFP, $\alpha$-fetoprotein; TNM, Tumor, Node and Metastasis.

transfection of miR-202 inhibitor promoted cell proliferation (Fig. 2C and D). Furthermore, the effects of increased miR-202 expression on cell glycolysis were detected in HCC cells. The present results suggested that glucose consumption was significantly decreased after 97-L and Huh7 cells were transfected with miR-202 mimic, compared with the control groups (Fig. 2E). Furthermore, lactate production was also decreased transfection with miR-202 mimic, compared with the control groups (Fig. 2F). The present results indicated that increased expression levels of miR-202 inhibited cell proliferation and cell glycolysis in HCC cells.

$H K 2$ is a direct target of miR-202. To uncover the molecular mechanisms underlying miR-202 expression affecting cell proliferation and cell glycolysis, the software miRanda was used to predict the potential targets of miR-202. Bioinformatics analysis revealed that HK2 was a potential target of miR-202 (Fig. 3A). Furthermore, the RT-qPCR results suggested that transfection of miR-202 mimic in $97 \mathrm{~L}$ and Huh7 cells could decrease the HK2 mRNA expression levels when compared with miR-NC groups (Fig. 3B). Consistent with the changes in HK2 mRNA expression levels, the protein expression 
A

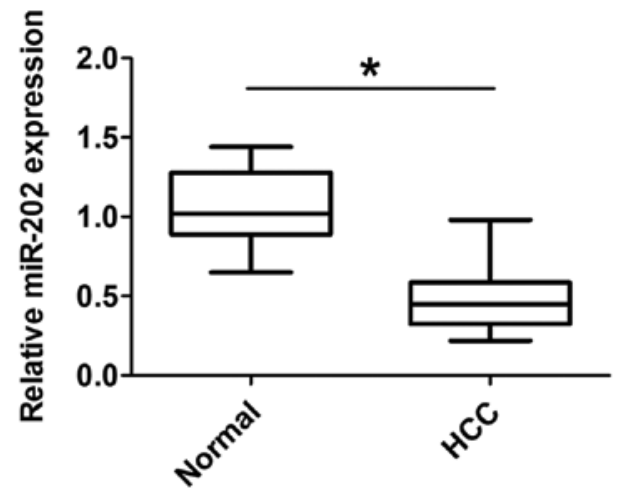

$\mathrm{B}$

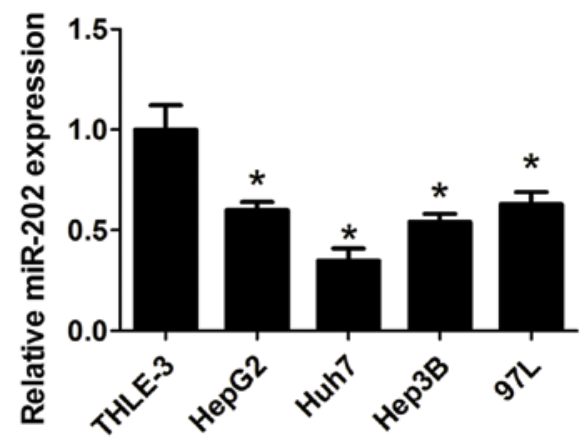

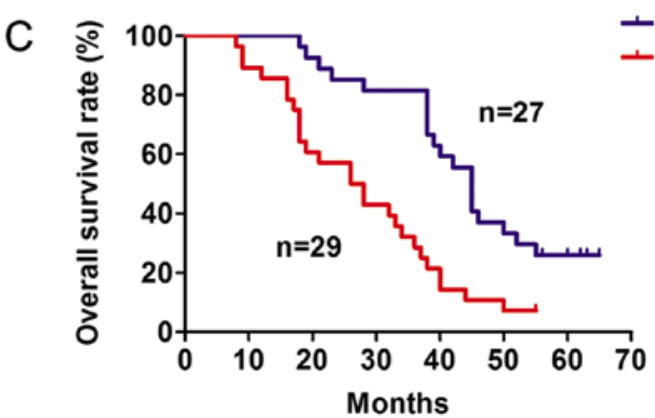

Figure 1. miR-202 expression is downregulated in HCC tissue samples and liver cancer cells. (A) Expression levels of miR-202 were determined in 56 paired human fresh HCC tissue and corresponding adjacent non-cancerous tissue samples using RT-qPCR assay. (B) Expression of miR-202 was detected using RT-qPCR assay in human liver cancer cells including Hep-G2, Hep3B, 97-L, Huh-7 and THLE-3 cells. (C) A survival plot was calculated using the Kaplan-Meier method and log-rank test assessing high miR-202 expression and low miR-202 expression groups. Data are presented as the mean \pm SD from three independent experiments. ${ }^{*} \mathrm{P}<0.05$ vs. corresponding control. miR, microRNA; HCC, hepatocellular carcinoma; RT-qPCR, reverse transcription-quantitative PCR.

A
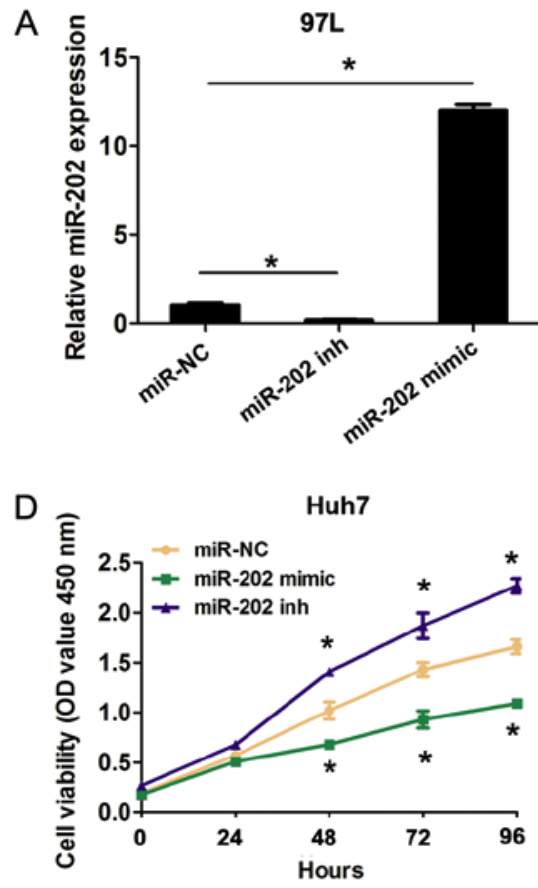

B

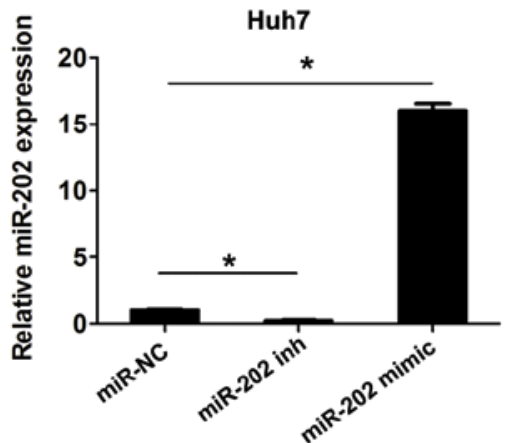

$\mathrm{E}$

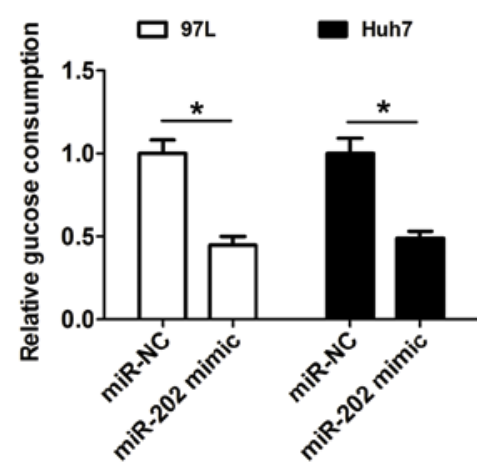

C

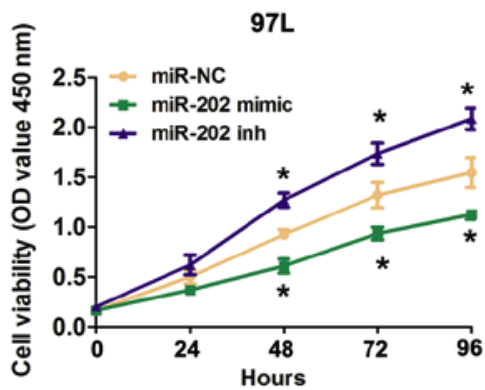

$\mathrm{F}$

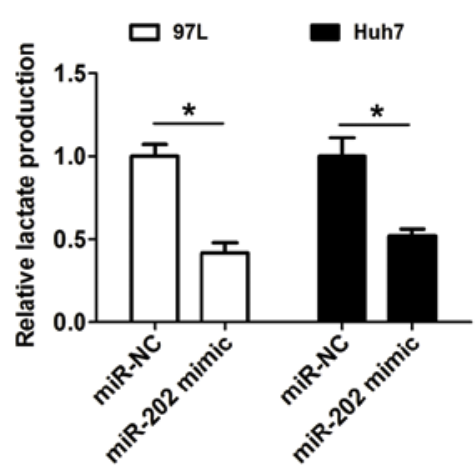

Figure 2. miR-202 inhibits cell proliferation and cell glycolysis in HCC. miR-202 expression was detected using reverse transcription-quantitative PCR following (A) 97-L and (B) Huh7 cell transfection with miR-NC, miR-202 mimic or miR-202 inhibitor. Cell Counting Kit 8 cell proliferation assay was used to evaluate cell proliferation following (C) 97-L and (D) Huh7 cell transfection with miR-NC, miR-202 mimic or miR-202 inhibitor. (E) Relative glucose consumption was revealed following 97-L and Huh7 cell transfection with miR-NC, miR-202 mimic or miR-202 inhibitor. (F) Relative lactate production was revealed following 97-L and Huh7 cell transfection with miR-NC, miR-202 mimic or miR-202 inhibitor. Data are presented as the mean \pm SD from three independent experiments. " $\mathrm{P}<0.05$ vs. corresponding control. miR, microRNA; HCC, hepatocellular carcinoma; NC, negative control; OD, optical density; inh, inhibitor. 
A

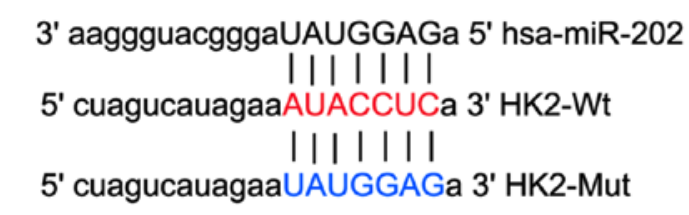

C

97L

GAPDH

HK2

Huh7
B
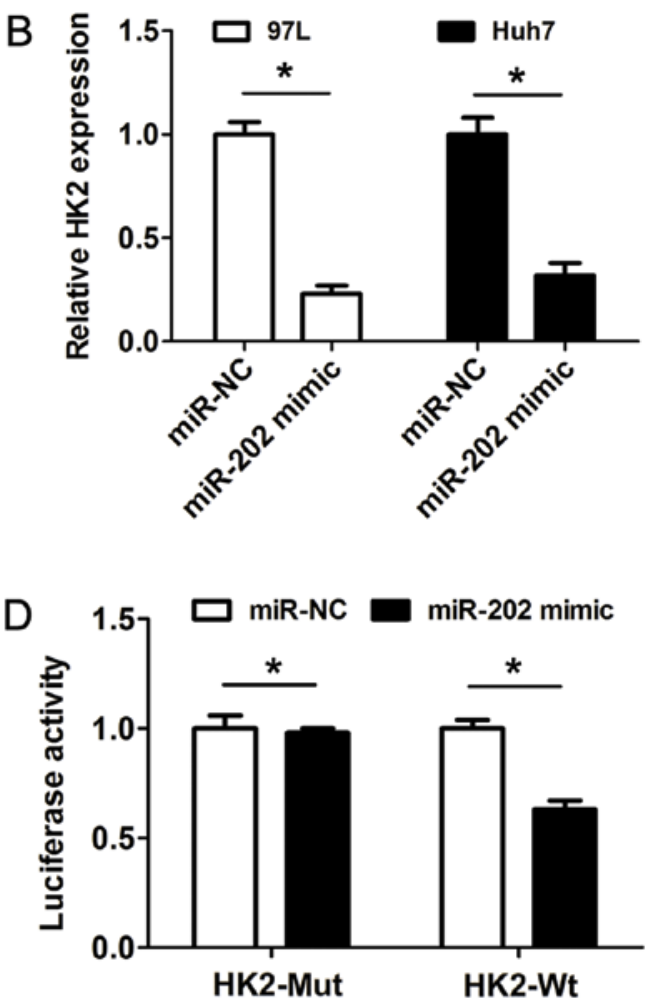

Figure 3. HK2 is a direct target of miR-202. (A) HK2 3'-UTR sequences containing the wild-type or mutated binding site of miR-202 were cloned into the psiCHECK2 vectors. (B) mRNA expression levels of HK2 following 97-L and Huh7 cell transfection with miR-NC or miR-202 mimic. (C) Protein expression levels of HK2 following 97-L and Huh7 cell transfection with miR-NC or miR-202 mimic. (D) Transfection of miR-202 mimic in 97 L cells markedly suppressed the luciferase activity of HK2 3'-UTR wild-type reporter construct, but not the mutant HK2 3'-UTR construct. Data are presented as the mean \pm SD from three independent experiments. ${ }^{*} \mathrm{P}<0.05$. HK2, hexokinase 2; 3'UTR, 3'untranslated region; WT, wild-type; Mut, mutant; NC, negative control.

levels of HK2 were also downregulated following miR-202 mimic transfection in $97 \mathrm{~L}$ and Huh7 cells, compared with the corresponding control groups (Fig. 3C). Furthermore, HK2 3'-untranslated region (3'UTR) sequences containing the wild-type or mutated binding site of miR-202 were cloned into the psiCHECK2 vectors, respectively (Fig. 3A). The dual-luciferase reporter gene assays revealed that transfection of miR-202 mimic in 97 L cells markedly suppressed the luciferase activity of the HK2 3'-UTR wild-type reporter construct, but not the mutant HK2 3'-UTR construct (Fig. 3D). Therefore, the present results suggested that HK2 was a direct target of miR-202.

miR-202 inhibits cell proliferation and cell glycolysis by targeting HK2 in HCC cells. To confirm the involvement of the $\mathrm{miR}-202 / \mathrm{HK} 2$ axis in the regulation of cell proliferation and cell glycolysis in HCC cells, HK2 was silenced using si-HK2 (Fig. 4A). The CCK8 cell proliferation assays revealed that knockdown of HK2 inhibited cell proliferation compared with the si-NC group, whereas co-transfection of miR-202 inhibitor plus si-HK2 reversed the inhibitory effects of HK2 silencing on cell proliferation (Fig. 4B). Furthermore, knockdown of HK2 inhibited cell glucose consumption and lactate production, while co-transfection of miR-202 inhibitor and si-HK2 reversed the inhibitory effects of HK2 knockdown on cell glucose consumption and lactate production (Fig. 4C and D). Therefore, the present results indicated that miR-202 inhibited cell proliferation and cell glycolysis by targeting HK2 in HCC cells.

\section{Discussion}

miR-202 has been described as a tumor-suppressing miRNA that is dysregulated in several different types of tumor. In a previous study, miR-202 was demonstrated to inhibit tumor progression by targeting laminin subunit $\alpha 1$ in esophageal squamous cell carcinoma (12). miR-202 inhibits the progression of human cervical cancer through inhibition of cyclin D1 (13). miR-202 functions as a tumor suppressor in non-small cell lung cancer by targeting STAT3 (14). miR-202 inhibits the cell proliferation, migration and invasion of glioma by directly targeting metadherin (15). In HCC, miR-202 was demonstrated to suppress cell proliferation by downregulating low-density lipoprotein receptor-related protein 6 post-transcriptionally (16). Therefore, the underlying molecular mechanism of miR-202 in the Warburg effect was investigated in the present study. The present results indicated that miR-202 expression was markedly downregulated in HCC tissue samples compared with the corresponding adjacent non-cancerous tissue samples. miR-202 expression levels were significantly associated with tumor size, vascular invasion and TNM stage. Furthermore, higher expression levels of miR-202 revealed a longer survival rate compared with lower levels of miR-202 expression. Therefore, the present results suggested that lower miR-202 may contribute to the development of HCC. However, the number of patients used in the present study was small, and so this sample size should be expanded in future studies.

Furthermore, the functional effects of miR-202 expression on HCC cells were detected in the present study. The results 

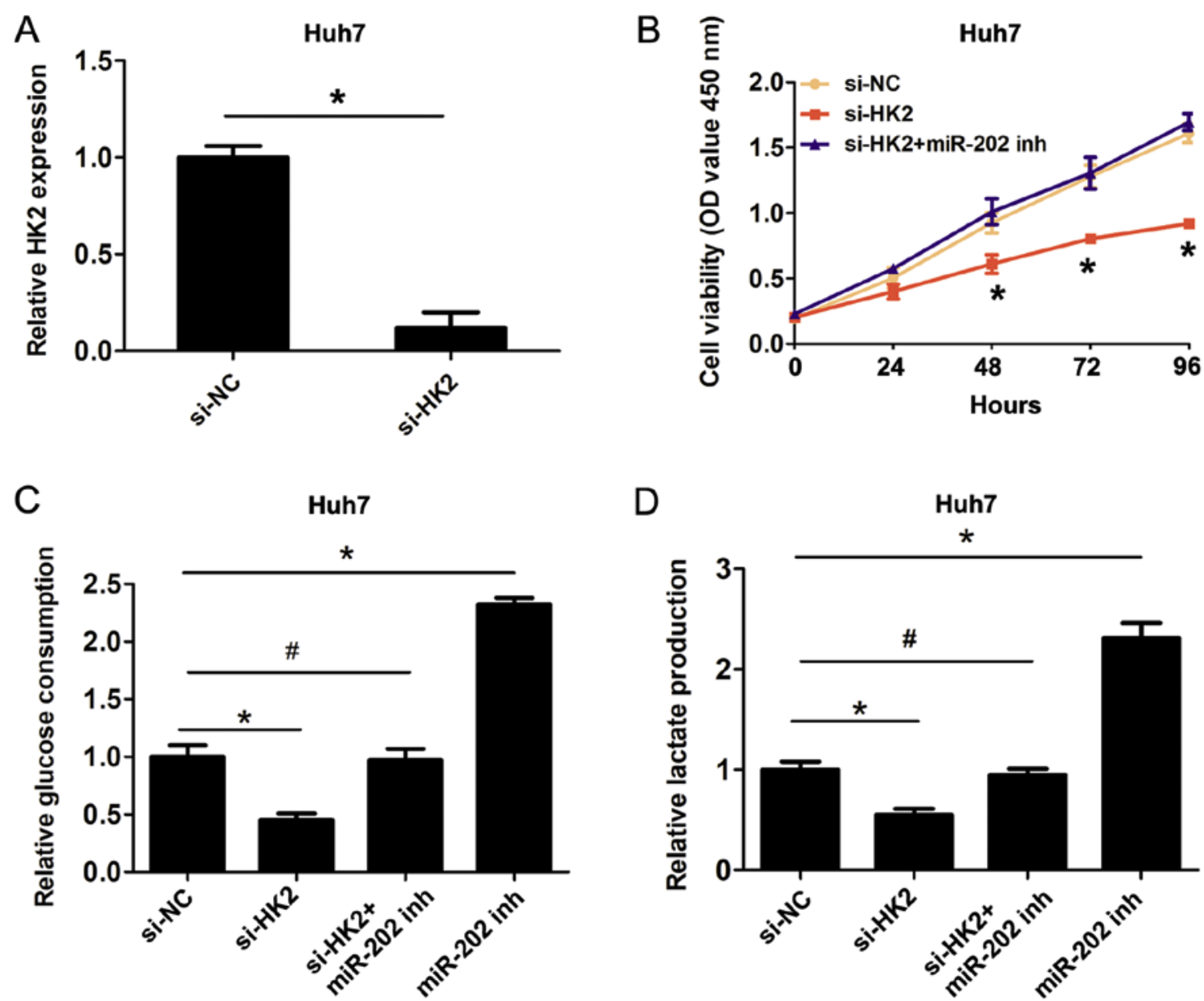

Figure 4. miR-202 inhibits cell proliferation and cell glycolysis by targeting HK2 in liver cancer cells. (A) Relative expression of HK2 following Huh7 cell transfection with si-NC or si-HK2. (B) Cell Counting Kit 8 cell proliferation assay was used to evaluate cell proliferation following Huh7 cell transfection with si-NC, si-HK2 or si-HK2 plus miR-202 inhibitor. (C) Relative glucose consumption and (D) lactate production were evaluated following Huh7 cell transfection with si-NC, si-HK2 miR-202 inhibitor or cotransfection with si-HK2 and miR-202 inhibitor. Data are presented as the mean \pm SD from three independent experiments. ${ }^{*} \mathrm{P}<0.05 ;{ }^{*} \mathrm{P}>0.05$. miR, microRNA; HK2, hexokinase 2; si, small interfering; NC, negative control; inh, inhibitor.

indicated that miR-202 could inhibit cell proliferation and cell glycolysis in HCC cells. The glycolytic enzyme HK2 is crucial for the Warburg effect, and elevated levels of HK2 had been revealed in a number of different types of human tumor, as well as being associated with cell glycolysis in tumor cells. As such, STAT3 regulates cell glycolysis via targeting HK2 in HCC (17). It has previously been demonstrated that miR-143 suppresses oral squamous cell carcinoma cell growth, invasion and glucose metabolism through targeting HK2 (18). forkhead box M1 promotes reprogramming of glucose metabolism in epithelial ovarian cancer cells via activation of GLUT1 and HK2 transcription (19). The miR-125a/HK2 axis regulates the reprogramming of cancer cell energy metabolism in HCC (20). In the present study, it was identified that HK2 was a direct target of miR-202 in HCC cells. miR-202 overexpression could decrease the HK2 mRNA and protein expression levels in HCC cells. Knockdown of HK2 inhibited cell proliferation, cell glucose consumption and lactate production, while co-transfection of miR-202 inhibitor and si-HK2 reversed the inhibitory effects of HK2 knockdown on cell glucose consumption and lactate production. Thus, these results indicated that miR-202 could inhibit cell proliferation and cell glycolysis by targeting HK2 in HCC cells.
Collectively, in the present study, the miR-202 expression levels were identified to be lower in HCC tissues and liver cancer cells. In addition, lower miR-202 expression levels were found to be associated with a poor prognosis in patients with HCC. Furthermore, miR-202 was found to function as a tumor suppressor in liver cancer cells by targeting HK2 to inhibit cell proliferation and cell glycolysis. Therefore, the present results suggested that miR-202 may represent a novel target for treating HCC.

\section{Acknowledgements}

Not applicable.

\section{Funding}

No funding was received.

\section{Availability of data and materials}

The datasets used and/or analyzed during the present study are available from the corresponding author upon reasonable request. 


\section{Authors' contributions}

JW, JC and FS conceived and designed the study, and drafted the manuscript. ZW, WX, YY, FD and HS collected, analyzed and interpreted the data, and critically revised the manuscript for intellectual content. All authors read and approved the final manuscript.

\section{Ethics approval and consent to participate}

The present study was approved by the Ethics Committee of Affiliated Hospital of Shaoxing University. Written informed consent was obtained from all patients in the study.

\section{Patient consent for publication}

Not applicable.

\section{Competing interests}

The authors declare that they have no competing interests.

\section{References}

1. Siegel R, Naishadham D and Jemal A: Cancer statistics, 2012 CA Cancer J Clin 62: 10-29, 2012.

2. Forner A, Llovet JM and Bruix J: Hepatocellular carcinoma. Lancet 379: 1245-1255, 2012.

3. Aravalli RN, Steer CJ and Cressman EN: Molecular mechanisms of hepatocellular carcinoma. Hepatology 48: 2047-2063, 2008.

4. Warburg O: On the origin of cancer cells. Science 123: 309-314, 1956.

5. Mathupala SP, Ko YH and Pedersen PL: Hexokinase-2 bound to mitochondria: Cancer's stygian link to the 'Warburg Effect' and a pivotal target for effective therapy. Semin Cancer Biol 19 : 17-24, 2009.

6. Singh PK, Mehla K, Hollingsworth MA and Johnson KR: Regulation of aerobic glycolysis by microRNAs in cancer. Mol Cell Pharmacol 3: 125-134, 2011.

7. Nie H, Li J, Yang XM, Cao QZ, Feng MX, Xue F, Wei L, Qin W, Gu J, Xia Q and Zhang ZG: Mineralocorticoid receptor suppresses cancer progression and the Warburg effect by modulating the miR-338-3p-PKLR axis in hepatocellular carcinoma. Hepatology 62: 1145-1159, 2015.

8. Zhang LF, Lou JT, Lu MH, Gao C, Zhao S, Li B, Liang S, Li Y, Li D and Liu MF: Suppression of miR-199a maturation by HuR is crucial for hypoxia-induced glycolytic switch in hepatocellular carcinoma. EMBO J 34: 2671-2685, 2015.
9. Wang B, Hsu SH, Frankel W, Ghoshal K and Jacob ST: Stat3-mediated activation of microRNA-23a suppresses gluconeogenesis in hepatocellular carcinoma by down-regulating glucose-6-phosphatase and peroxisome proliferator-activated receptor gamma, coactivator 1 alpha. Hepatology 56: 186-197, 2012.

10. Livak KJ and Schmittgen TD: Analysis of relative gene expression data using real-time quantitative PCR and the 2(-Delta Delta C(T)) Method 25: 402-408, 2001.

11. Zhong JH, Xiang BD, Gong WF, Ke Y, Mo QG, Ma L, Liu X and Li LQ: Comparison of long-term survival of patients with BCLC stage B hepatocellular carcinoma after liver resection or transarterial chemoembolization. PLoS One 8: e68193, 2013.

12. Meng X, Chen X, Lu P, Ma W, Yue D, Song L and Fan Q: MicroRNA-202 inhibits tumor progression by targeting LAMA1 in esophageal squamous cell carcinoma. Biochem Biophys Res Commun 473: 821-827, 2016.

13. Yi Y, Li H, Lv Q, Wu K, Zhang W, Zhang J, Zhu D, Liu Q and Zhang W: miR-202 inhibits the progression of human cervical cancer through inhibition of cyclin D1. Oncotarget 7: 72067-72075, 2016.

14. Zhao Z, Lv B, Zhang L, Zhao N and Lv Y: miR-202 functions as a tumor suppressor in non-small cell lung cancer by targeting STAT3. Mol Med Rep 16: 2281-2289, 2017.

15. Yang J, Fan B, Zhao Y and Fang J: MicroRNA-202 inhibits cell proliferation, migration and invasion of glioma by directly targeting metadherin. Oncol Rep 38: 1670-1678, 2017.

16. Zhang Y, Zheng D, Xiong Y, Xue C, Chen G, Yan B and Ye Q: miR-202 suppresses cell proliferation in human hepatocellular carcinoma by downregulating LRP6 post-transcriptionally. FEBS Lett 588: 1913-1920, 2014.

17. Li M, Jin R, Wang W, Zhang T, Sang J, Li N, Han Q, Zhao W, Li C and Liu Z: STAT3 regulates glycolysis via targeting hexokinase 2 in hepatocellular carcinoma cells. Oncotarget 8: 24777-24784, 2017.

18. Sun $X$ and Zhang L: MicroRNA-143 suppresses oral squamous cell carcinoma cell growth, invasion and glucose metabolism through targeting hexokinase 2. Biosci Rep 37: pii: BSR20160404, 2017.

19. Wang Y, Yun Y, Wu B, Wen L, Wen M, Yang H, Zhao L, Liu W, Huang S, Wen N and Li Y: FOXM1 promotes reprogramming of glucose metabolism in epithelial ovarian cancer cells via activation of GLUT1 and HK2 transcription. Oncotarget 7: 47985-47997, 2016.

20. Jin F, Wang Y, Zhu Y, Li S, Liu Y, Chen C, Wang X, Zen K and $\mathrm{Li} \mathrm{L}$ : The miR-125a/HK2 axis regulates cancer cell energy metabolism reprogramming in hepatocellular carcinoma. Sci Rep 7: 3089, 2017. International (CC BY-NC-ND 4.0) License. 